\title{
La Convention européenne des droits de l'homme, rempart contre les remparts opposés aux migrants
}

The European Convention on Human Rights, a Safeguard against Anti-Migrant Laws

El convenio europeo de los derechos humanos: un escudo contra aquellos que ponen trabas a los derechos de los migrantes

\section{Céline Lageot}

\section{OpenEdition}

\section{Journals}

Édition électronique

URL : https://journals.openedition.org/remi/9017

DOI : $10.4000 /$ remi. 9017

ISSN : $1777-5418$

Éditeur

Université de Poitiers

Édition imprimée

Date de publication : 1 décembre 2017

Pagination : 197-203

ISBN : 979-10-90426-60-3

ISSN : 0765-0752

\section{Référence électronique}

Céline Lageot, « La Convention européenne des droits de l'homme, rempart contre les remparts opposés aux migrants », Revue européenne des migrations internationales [En ligne], vol. 33 - n4 | 2017, mis en ligne le 01 décembre 2019, consulté le 14 avril 2022. URL : http://journals.openedition.org/ remi/9017; DOI : https://doi.org/10.4000/remi.9017 


\section{Chronique juridique}

\section{La Convention européenne des droits de l'homme, rempart contre les remparts opposés aux migrants}

\section{Céline Lageot ${ }^{1}$}

Depuis fort longtemps, les hommes traversent de vastes territoires pour venir s'installer en Europe. Certains d'entre eux viennent même y chercher une protection internationale. C'est ce que I'on appelle les demandeurs d'asile. Or, il n'est pas toujours aisé de comprendre la différence effectuée entre migrants, demandeurs d'asile et réfugiés, principalement du fait de l'utilisation impropre de ces termes par les médias. Les " migrants " correspondent à cette catégorie de personnes qui se déplacent d'un endroit, d'une région ou d'un pays à un autre et ce pour quelle que raison que ce soit, y compris pour un motif économique. Le terme de " demandeur d'asile " est plus précis et plus juridique quant à lui, puisqu'il vise un migrant qui demande à pouvoir bénéficier de la protection internationale.

Or, en Europe, la protection internationale peut découler du statut de réfugié ou de la protection dite subsidiaire.

Le statut de réfugié est envisagé par la Convention de Genève de 1951 qui porte précisément sur cette question. L'État qui a ratifié cette convention, comme la France par exemple le 23 juin 1954, peut reconnaître le statut de réfugié à toute personne qui nourrirait des craintes fondées d'être persécutée, dans son pays d'origine et ce, en raison de sa race, de sa religion, de sa nationalité, de son appartenance à un groupe social particulier ou de ses opinions politiques.

Deuxième hypothèse, si un État considère qu'un migrant devrait se voir protégé, mais pour des raisons qui ne figurent pas dans la Convention de Genève sur le statut des réfugiés, il peut alors décider d'accorder la protection subsidiaire. Autrement dit, la protection juridique internationale dont peuvent bénéficier les migrants est directement liée au motif de leur déplacement. S'ils fuient leur pays pour échapper à la persécution au sens de l'article premier de la Convention de 1951, ce sont des "demandeurs d'asile " ou des "réfugiés", et ils pourront alors prétendre à cette protection spéciale accrue et garantie par ce traité international. S'ils quittent leur pays pour toute autre raison, ils seront

1 Professeur de droit public, Université de Poitiers, Faculté de droit et des sciences sociales, CECOJI, 2 rue Jean Carbonnier, Bâtiment A1, TSA 81100, 86073 Poitiers Cedex ; celine.lageot@univ-poitiers.fr 
alors considérés comme des migrants. Ils ne jouiront pas, de ce fait, d'une protection spécifique en vertu de la Convention de Genève, mais d'une protection générale en vertu du droit international des droits de I'homme.

La protection internationale n'est malheureusement pas de la compétence de la Cour européenne des droits de l'homme, puisque la Cour de Strasbourg n'a pas la possibilité d'examiner l'application de la Convention de Genève.

Au niveau de la protection européenne, la Convention européenne des droits de l'homme du 4 novembre 1950 ne garantit pas non plus expressément de droit à l'asile. Le contrôle des flux migratoires est, en effet et en principe, de la compétence souveraine des États. Par conséquent, le contrôle de l'entrée, du séjour et le cas échéant de l'expulsion des étrangers est du ressort des États souverains. Cependant, et cette fois-ci fort heureusement, les États membres du Conseil de I'Europe et de la Convention européenne des droits de l'homme ont l'obligation de garantir à toute personne relevant de leur juridiction, y compris aux migrants donc, le respect des droits envisagés par la Convention de 1950. C'est ce que prévoit très clairement l'article 1 de la Convention de 1950. C'est donc à cette fin que la jurisprudence de la Cour de Strasbourg impose certaines limitations aux droits des États de refuser l'accès à leurs frontières².

Mises à part ces décisions de la Cour de Strasbourg portant sur ce point précis du refus à l'accès au territoire, nous nous proposons d'abord de présenter synthétiquement quels droits de la Convention européenne des droits de I'homme peuvent faire obstacle à l'éloignement de migrants demandeurs d'asile.

\section{Les droits de la Convention pouvant servir de bouclier contre l'éloignement de migrants demandeurs d'asile}

En premier lieu, il y a les articles 2 et 3 de la Convention, le premier garantissant le droit à la vie, le second interdisant la torture et les traitements inhumains ou dégradants. Aucun individu ne peut être éloigné vers un territoire où il encourrait un risque avéré pour sa vie. C'est le principe de non-refoulement aux frontières pour des raisons pouvant porter atteinte à la vie d'un individu ou à son intégrité physique ou morale qui est ici activé par la Cour de Strasbourg. Par exemple, dans une affaire de 2011 impliquant le Royaume-Uni, la Cour a constaté que I'expulsion des deux requérants en Somalie aurait emporté violation de I'article 3, eu égard à la crise humanitaire et à la violence aveugle qui prévalaient à Mogadiscio à l'époque des faits ${ }^{3}$.

Les articles 2 et 3 de la Convention peuvent aussi s'appliquer lorsqu'est refusée aux frontières l'entrée sur le territoire de personnes en danger, ou interceptées en mer. Ainsi, la Cour a jugé en 2012 qu'un groupe de migrants, qui avaient été interceptés en pleine mer par les autorités italiennes, n'auraient pas

\footnotetext{
2 Abdulaziz, Cabales et Balkandali c. Royaume-Uni, 9214/80, 28 mai 1985, § 67, série A n 94 et Saadi c. Italie [GC], 37201/06, §§ 124-125, CEDH 2008.

3 Sufi et Elmi c. Royaume-Uni, 8319/07 et 11449/07, 28 juin 2011.
} 
dû être sommairement renvoyés en Libye où ils ont dû faire face aux risques avérés de traitements contraires à l'article 3 . Au lieu de cela, a ajouté la Cour, il aurait fallu prévoir la possibilité pour ces migrants de demander l'asile en Italie ${ }^{4}$.

D'autres dispositions de la Convention européenne peuvent aussi jouer comme bouclier contre l'éloignement des migrants demandeurs d'asile. Ce sont les articles 5 et 6 de la Convention, le premier prévoyant le droit à la liberté et à la sureté, soit le droit de ne pas être arrêté et détenu arbitrairement, le second fixant le principe du droit à un procès équitable. Ces dispositions peuvent être activées dans I'hypothèse où l'État de destination aurait arbitrairement placé en détention un individu, sans jugement préalable, ou l'aurait emprisonné pendant une longue période, à la suite d'une condamnation prononcée à l'issue d'un procès manifestement inéquitable. Néanmoins, un seuil très élevé s'applique dans de telles hypothèses. Dans une affaire concernant l'expulsion du Royaume-Uni vers la Jordanie, la Cour a constaté, par exemple, que le fait pour une personne d'avoir été mise en détention provisoire durant cinquante jours restait bien en deçà de la durée de détention pouvant entraîner une violation évidente de l'article 5. En outre, I'admission de preuves, obtenues sous la torture dans le cadre d'un nouveau procès pénal, représenterait un flagrant déni de justice, en violation de l'article 6 de la Convention ${ }^{5}$. Cette décision a continué à entretenir la rébellion grandissante du RU contre la Cour de Strasbourg depuis les décisions sur le droit de vote des détenus de 2005.

\section{Une appréciation particulièrement exigeante de la Cour de Strasbourg concernant l'article 3 (interdiction de la torture, des traitements inhumains ou dégradants)}

Pour constituer une violation de l'article 3 de la Convention, la Cour européenne des droits de l'homme exige en premier lieu que le traitement atteigne un seuil minimum de gravité, qui s'apparente le plus souvent au traitement dégradant ${ }^{6}$. L'appréciation de ce seuil par la Cour dépend de l'ensemble des circonstances de l'espèce, notamment de l'âge du requérant, de son sexe, de son état de santé, de son appartenance à une minorité, etc. ${ }^{7}$. Tous ces éléments sont examinés cumulativement par la Cour. Toute appréciation par la Cour de Strasbourg, aux fins de déterminer si un demandeur d'asile est susceptible de subir un traitement contraire à l'article 3, est de ce fait individualisée et fondée sur l'ensemble des éléments disponibles. Conformément à la jurisprudence de la Cour de Strasbourg, le risque de mauvais traitements dans le pays de destination doit être " réel ", "prévisible " et " personnel ". C'est pourquoi la Cour exigera que le requérant migrant prouve l'existence de circonstances spécifiques qui le rendrait particulièrement et personnellement vulnérable. Ces circonstances spécifiques peuvent être constituées à partir de preuves concernant de précédents mauvais traitements subis par la population dans le pays

\footnotetext{
4 Hirsi Jamaa et autres c. Italie [GC], 27765/09, CEDH 2012.

5 Othman (Abu Qatada) c. Royaume-Uni, 8139/09, CEDH 2012.

6 Soering c. Royaume-Uni, 14038/88, 7 juillet 1989, série A n 161, § 100.

7 Ireland c. Royaume-Uni, 5310/71, 18 janvier 1978, série A nº 25.
} 
de destination, par l'octroi dans le passé du statut de réfugié par des États aux demandeurs d'asile de ce pays de destination, ou par différentes évaluations ou rapports émis par le Haut-Commissaire des Nations Unies pour les réfugiés ${ }^{8}$.

Ces circonstances peuvent être aussi démontrées dès lors de l'existence de persécutions systématiques de personnes se trouvant dans une situation analogue, sous réserve pour la Cour que ce groupe soit identifiable. Par exemple, la Cour a rendu une décision en ce sens en ce qui concerne les membres de la minorité Ashraf en Somalie ${ }^{9}$.

La Cour a aussi reconnu que l'exposition d'un individu à une situation de violence générale d'une intensité exceptionnelle peut être suffisante pour conclure que la personne court le risque d'être victime de mauvais traitements, simplement du fait de sa présence dans la zone en question ${ }^{10}$.

Par ailleurs, dans certaines circonstances, exposer un demandeur d'asile à des conditions extrêmes de pauvreté peut aussi constituer une violation de I'article $3^{11}$. La Cour a ainsi constaté que les conditions de vie dans les principaux camps de réfugiés au Kenya et en Somalie, en particulier le grave surpeuplement et l'accès très limité aux abris, à l'eau et aux installations d'assainissement, étaient si désastreuses, qu'elles atteignaient le seuil minimal de gravité de déclenchement de I'application de I'article $3^{12}$.

En tout état de cause, il appartiendra en principe au requérant de produire des éléments propres à démontrer qu'il y a des raisons sérieuses de penser que, s'il était renvoyé d'un État membre du Conseil de l'Europe, il serait exposé à un risque réel de mauvais traitements dans le pays de destination, considéré alors comme un État "non sûr ". Lorsque de tels éléments sont avancés par un requérant, c'est alors au Gouvernement de dissiper tout doute éventuel ${ }^{13}$.

Mais la Cour a reconnu que les migrants demandeurs d'asile se trouvent souvent dans une situation spéciale de très grand dénuement, exigeant alors qu'on accorde davantage crédit à leurs déclarations qu'aux documents à l'appui de leurs prétentions, ceux-ci faisant très souvent défaut ${ }^{14}$.

Les affaires introduites devant la Cour sont toujours examinées au regard de la situation individuelle de chaque requérant. Mais certains requérants peuvent appartenir à des groupes particulièrement vulnérables par nature et la Cour a reconnu que, pour cette raison, ils ont besoin d'une protection spécifique ${ }^{15}$.

8 Singh et autres c. Belgique, 33210/11, 2 octobre 2012.

9 Salah Sheekh c. Pays-Bas, 1948/04, 11 janvier 2007 et, a contrario, Vilvarajah et autres c. Royaume-Uni, 13163/87 et autres, 30 octobre 1991, série A n²15.

10 Sufi et Elmi c. Royaume-Uni, 8319/07 et 11449/07, 28 juin 2011.

11 M.S.S. c. Belgique et Grèce [GC], 30696/09, CEDH 2011.

12 Sufi et Elmi c. Royaume-Uni, 8319/07 et 11449/07, 28 juin 2011.

13 Saadi c. Italie [GC], 37201/06, CEDH 2008.

14 Salah Sheekh c. Pays-Bas, 1948/04, 11 janvier 2007 et R.C c. Suède, 41827/07, 9 mars 2010.

15 M.S.S. c. Belgique et Grèce [GC], 30696/09, CEDH 2011. 


\section{La prise en compte par la Cour de Strasbourg de l'existence de groupes particulièrement vulnérables}

Ces groupes vulnérables peuvent s'apparenter à des minorités qui sont systématiquement soumises à de mauvais traitements dans un pays ou une région donnés, ou à certaines catégories - les enfants, les femmes, les femmes enceintes, les personnes handicapées ou les personnes âgées - reconnues comme ayant des besoins spécifiques.

Or, une importance particulière est attachée au statut de requérant demandeur $d^{\prime}$ asile. Il en est ainsi parce qu'un large consensus existe au niveau international et européen selon lequel les demandeurs d'asile représentent un groupe particulièrement défavorisé et vulnérable et qui a par conséquent besoin d'une protection spécifique.

L'appartenance d'un requérant à un groupe particulièrement vulnérable peut avoir un impact sur les obligations imposées aux États, quant aux modalités d'accueil de la personne et concernant la question de savoir si elle peut ou non fait l'objet d'une mesure d'éloignement d'un État membre du Conseil de I’Europe.

La situation des mineurs non accompagnés ou des mineurs isolés demandant asile revêt une importance toute particulière de ce point de vue là. Cette problématique a été notamment traitée par la Cour de Strasbourg dans l'emblématique affaire Rahimi, soit ce jeune homme âgé de quinze ans qui venait d'Afghanistan et qui cherchait asile en Grèce ${ }^{16}$. La Cour a constaté que les conditions dans lesquelles ce mineur non accompagné a été initialement détenu, puis l'échec ultérieur des autorités grecques à s'occuper de lui après sa sortie du centre de rétention, ont constitué un traitement dégradant, tout à fait contraire à l'article 3 de la Convention.

Dans le sillage des exigences conventionnelles posées par l'article 3 et la prise en compte de groupes de personnes particulièrement vulnérables, la Cour de Strasbourg en déduit encore des obligations positives pesant à la charge des États.

\section{Les conditions d'accueil des demandeurs d'asile : des obligations positives à la charge des États}

Selon la Cour de Strasbourg, I'article 3 de la Convention exige en effet que les États d'accueil fournissent un logement et des conditions matérielles d'accueil convenables aux demandeurs d'asile, ces derniers arrivant appauvris et complètement dépendants de l'assistance de l'État d'accueil.

Dans son arrêt de principe sur la question ${ }^{17}$, la Cour a constaté que la Grèce n'avait pas respecté ses obligations au titre de l'article 3, dès lors qu'elle $n$ 'avait pas garanti au requérant des conditions d'accueil décentes, alors que sa

16 Rahimi c. Grèce, 8687/08, 5 avril 2011.

17 M.S.S. c. Belgique et Grèce [GC], 30696/09, CEDH 2011. 
procédure d'asile était en suspens. Quelles circonstances ont été particulièrement significatives pour la Cour et la conduire ainsi à une telle conclusion ? En premier lieu, le grave surpeuplement et les conditions sanitaires très dégradées dans le centre d'accueil des migrants où le requérant a été initialement détenu. En deuxième lieu, le fait que, après sa sortie, le requérant a été contraint à vivre pendant de nombreux mois dans un parc, dans un état de pauvreté extrême, incapable de subvenir à la plupart de ses besoins élémentaires. En troisième lieu enfin, le fait que la situation du requérant s'était encore aggravée par sa crainte permanente d'être attaqué et volé, et par l'absence de perspectives d'amélioration de sa situation.

Dans une autre affaire de ce type introduite cette fois-ci contre la Suisse ${ }^{18}$, la Cour a constaté que les conditions d'accueil pour les demandeurs d'asile en Italie étaient certes loin d'être aussi problématiques qu'en Grèce, et qu'elles ne pouvaient donc constituer en elles-mêmes un obstacle à tout éloignement des demandeurs d'asile vers ce pays, ce que souhaitait la Suisse. Cependant, la Cour a considéré que les requérants se trouvaient dans une situation tout à fait spécifique, dès lors qu'ils formaient une famille de huit personnes avec six enfants mineurs, dont un enfant en bas âge. Elle a donc conclu que, même en I'absence de lacunes systémiques en Italie, contrairement à la Grèce, il incombait aux autorités de l'État de renvoi (en l'occurrence la Suisse) d'obtenir des assurances de la part des autorités du pays de destination (en l'occurrence l'Italie), qu'à leur arrivée, les demandeurs seraient reçus dans des structures et dans des conditions adaptées à l'âge des enfants, et que la famille ne serait pas séparée.

En guise de conclusion, on retiendra que les États membres du Conseil de l'Europe sont pleinement souverains pour décider quels demandeurs d'asile sont éligibles au bénéfice de la protection internationale. Et de ce fait, il n'appartient pas à la Cour de Strasbourg de décider du fond des demandes d'asile individuelles. Cependant, dans l'exercice du contrôle de leurs frontières et des flux migratoires, les États doivent néanmoins agir conformément aux normes établies dans la Convention européenne des droits de l'homme et aux principes découlant du vaste corpus jurisprudentiel de la Cour de Strasbourg, et ce afin de garantir le respect des droits les plus fondamentaux des demandeurs d'asile. Avant d'être des demandeurs d'asile, des migrants, des étrangers, ils n'en sont pas moins des hommes, à qui doivent être reconnus des droits, naturels, imprescriptibles, universels, juste - mais c'est déjà beaucoup - parce qu'ils sont hommes.

18 Tarakhel c. Suisse [GC], 29217/12, CEDH 2014. 


\section{Références bibliographiques}

Basilien-Gainche Marie-Laure (2017) Les frontières européennes. Quand le migrant incarne la limite, Revue de I'Union européenne, 609, pp. 61-84.

Cuttitta Paolo (2007) Le monde-frontière. Le contrôle de l'immigration dans I'espace globalisé, Cultures \& Conflits, 68, pp. 61-84.

Foegle Jean-Philippe (2017) Un renforcement en trompe-l'œil des droits des migrants retenus aux portes de l'Europe, La Revue des Droits de l'Homme, Actualités Droits-Libertés, [en ligne]. URL : https://journals.openedition.org/ $\mathrm{revdh} / 2904$

Imbert Louis, Kamoun Sara et Laborde Pauline (2016) Un rapport emblématique de l'actualité migratoire et de l'architecture évolutive de la protection des droits, La Revue des Droits de I'Homme, Actualités Droits-Libertés, [en ligne], consulté le 30 avril 2018. URL : http://journals.openedition.org/revdh/2858

Klötgen Paul (2010) La frontière et le droit. Esquisse d'une problématique, in Jean-Luc Deshayes et Didier Francfort Dirs., Du barbelé au pointillé : les frontières au regard des sciences humaines et sociales, Nancy, Presses Universitaires de Nancy, pp. 131-158.

Lageot Céline (2016) Les enseignements de I'affaire Tarakhel: le raisonnement enrichi des juges à la source d'une protection renforcée des migrants en Europe, R.T.D.H., 1, pp. 245-260.

Parrot Karine et Imbert Louis (2016) La " crise migratoire " fantasmée ou l'échec programmé de la forteresse Europe, Revue des Juristes de Sciences Po, 12, pp. 49-62. 